\title{
Fertility and Pregnancy Outcomes after High-Intensity Focused Ultrasound Ablation for Uterine Fibroids and Adenomyosis: A Review
}

\author{
Authors: \\ *Kwok To Thomas Li, ${ }^{1}$ WS Felix Wong ${ }^{2}$ \\ 1. Department of Obstetrics and Gynaecology, Queen Mary Hospital, The University \\ of Hong Kong, China \\ 2. Division of Women's and Children's Health, The University of New South Wales, \\ Sydney, Austraila \\ *Correspondence to lithomas8@gmail.com
}

Disclosure: The authors have declared no conflicts of interest.

Received:

28.03.21

Accepted:

22.10 .21

Keywords:

Adenomyosis, high-intensity focused ultrasound (HIFU), pregnancy, uterine fibroid.

Citation:

EMJ Innov. 2021;6[1]:50-54.

\section{Abstract}

High-intensity focused ultrasound (HIFU) ablation, also known as focused ultrasound surgery, is the latest advancement in treating uterine fibroids and adenomyosis. Limited evidence, in terms of case series only, suggest that ultrasound-guided focused ultrasound/magnetic resonance-guided focused ultrasound treatment for fibroids and adenomyosis could be a safe alternative to myomectomy or uterine artery embolisation for females who wish to have babies. It also appears that this non-invasive HIFU treatment can shorten the treatment to pregnancy interval after HIFU ablation. Further studies are needed to confirm such findings.

\section{INTRODUCTION}

Uterine fibroids and adenomyosis are common among reproductive-age females. Uterine fibroids, particularly large submucosal and intramural uterine fibroids, may cause infertility and implantation and miscarriage problems.,2 They may also increase the risk of complications during pregnancy and delivery; during pregnancy, they may cause inappropriate enlargement of the uterus, fetal malpresentation, obstructed labour, caesarean delivery, and postpartum haemorrhage. ${ }^{3,4}$

Adenomyosis, though arising from different aetiology, may also cause similar problems. Furthermore, adenomyosis can interfere with the

process of pregnancy by disrupting implantation. ${ }^{5}$ They are also the most common cause for heavy menstrual bleeding, leading to anaemia before contemplating pregnancy. ${ }^{6}$ Medical treatments with hormones or progestogen intrauterine contraceptive devices prevent these patients from conception and pregnancy. Therefore, surgical treatment is the usual resort to improve their chance of pregnancy. ${ }^{7}$

\section{METHODOLOGY}

Methodology of this study of high-intensity focused ultrasound (HIFU) ablation involved literature search using PubMed as the search engine and using keywords such as 'highintensity focused ultrasound', 'HIFU', 'fibroid', 
'adenomyosis', and 'pregnancy' from 2001 to 2021. Additional literature searches were conducted from peer-reviewed colleges' publications and guidelines, as well as of references from the co-author's books: Adenomyosis Facts and Treatments by Xue et al. ${ }^{8}$ and Focused UItrasound Surgery in Gynecology by Wong et al. ${ }^{9}$

\section{WHAT IS HIGH-INTENSITY FOCUSED ULTRASOUND AND HOW IT WILL HELP TO IMPROVE FERTILITY?}

HIFU ablation, also known as focused ultrasound surgery (FUS), is the latest advancement in treating many benign solid tumours in gynaecology and surgical conditions, ${ }^{10-14}$ including, but not limited to, uterine fibroids and

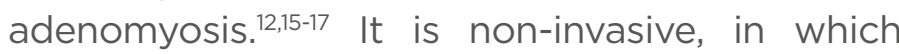
the most striking feature is no cut wound of the uterine wall in which the lesions are located.

HIFU focuses the ultrasound energy on the target lesions such as uterine fibroids or adenomyosis without affecting the surrounding organs. The localised temperature to the target lesion is $60-90{ }^{\circ} \mathrm{C}$. The surrounding tissue and vessels under lower temperature dissipate the heat by their blood flow. Therefore, the damage to the surrounding tissue and vessels could be minimised when the HIFU heat is applied in a pulsed manner. This induces coagulation necrosis of the uterine fibroids or adenomyosis without damage to the surrounding organs, particularly the major vessels. Subsequently, the necrotic fibroid or adenomyosis tissues are absorbed and removed, leading to reduced sizes of the treated lesions.

After HIFU treatment, either with the use of ultrasound or MRI to assess the size of fibroids, most of the fibroids reduce at least $50.0 \%$ in size after a single treatment. Lyon et al. ${ }^{18}$ reported a case series of 10 patients, with a $23.3 \%$ reduction in size at 3 months, $49.3 \%$ at 12 months, and $51.9 \%$ at 24 months. A retrospective case series of 189 nulliparous females also showed the mean reduction in uterine fibroids volume was $58.0 \% \pm 31.3 \%$ in 12 months after HIFU ablation. ${ }^{19}$ These reductions in uterine sizes greatly enhance the fertility environment of the uterus for pregnancy. HIFU also has a strong advantage in maintaining the integrity of the uterus. Using ultrasound focusing on ablating adenomyosis lesions can restore the uterus's anatomy and improve the immune microenvironment; the pregnancy rate increased after HIFU treatment for adenomyosis. ${ }^{20}$

\section{HIGH-INTENSITY FOCUSED ULTRASOUND TECHNIQUE}

The target adenomyosis tissue is identified through visualisation by comparing ultrasound and MRI images during HIFU treatment. HIFU ablation is performed to ablate the adenomyosis tissue point by point, layer by layer, until greyscale changes of the coagulated tissue are obvious. Under MRI, it is not too difficult after to identify the irregular boundary between adenomyosis and normal myometrium. Improving static ultrasound technology helps to identify adenomyosis; also, using cine clips (movie editing function) if available can easily diagnose adenomyosis. ${ }^{8}$

\section{HIGH-INTENSITY FOCUSED ULTRASOUND ABLATION COMPARED WITH MYOMECTOMY OR ADENOMYOMECTOMY}

Myomectomy or adenomyomectomy are also performed on patients who wish to retain their uterus, but fibroid recurrence rates after abdominal myomectomy are $12.4 \%$ and $46.0 \%$ at 12 and 24 months, respectively. ${ }^{21}$ The need for re-operation for recurrent symptoms in adenomyosis is high. The risk of recurrence of uterine fibroid after HIFU is $15 \%$, which is lower or at least comparable with myomectomy. These surgical procedures are also associated with morbidity or even mortality.

HIFU ablation is a new technology. Its safety profile is best-known in treating spaceoccupying lesions in gynaecology. Furthermore, the reported complications are minor. Chen et al. ${ }^{22}$ demonstrated in a large cohort study that complications arising from HIFU ablation were all Grade A or B but not C or above, according to the Society of Interventional Radiology (SIR) Severity Classification standard.23 In another safety analysis of 9,988 cases of uterine fibroids and adenomyosis treated by HIFU ablation, Chen et al. ${ }^{24}$ noted that more serious complications occurred when treating 
adenomyoma, reflecting the difference in the pathology of the two different types of disease. However, the incidence of complications in HIFU ablation is comparatively low. ${ }^{25}$

After HIFU ablation, improvement of symptoms and quality of life were significantly better than surgical myomectomy. ${ }^{26}$ The improvement of haemoglobin level enhances successful pregnancy and delivery outcomes.

After myomectomy, patients who may become pregnant have risks of uterine rupture, miscarriage, pre-term birth, and intrauterine adhesions that impair pregnancy. ${ }^{27}$ These patients are also advised not to get pregnant within 1-2 years to allow the uterine wound to heal well before pregnancy. Even if previous surgery had not affected the chance of pregnancy, the mode of delivery is still controversial. When myomectomy, or uncommonly adenomyomectomy, has been performed on a female with fibroids or adenomyosis, some obstetricians may allow a trial of a vaginal birth if there is no endometrial opening of the uterine cavity during myomectomy. However, some doctors in some Asian countries, because of the unavailable detailed history of previous myomectomy or adenomyomectomy, may choose to offer elective caesarean section to avoid risk of uterine rupture during labour.

\section{COMPARISON WITH UTERINE ARTERY EMBOLISATION}

HIFU ablation is a non-invasive procedure that has often been compared with uterine artery embolisation (UAE), which has also been accepted as an effective alternative treatment for uterine fibroids and adenomyosis instead of surgery. 28,29 However, regarding fertility, pregnancy, and delivery outcomes, major complications include uterine necrosis and infection leading to emergent hysterectomy, ovarian failure, amenorrhoea, and vaginal dryness related to non-target embolisation or over-embolisation. ${ }^{30,31}$ At present, there is no definite conclusion about the safety of pregnancy after UAE. There are reports of cases of successful pregnancy and delivery after UAE; however, adverse outcomes of pregnancy after UAE including spontaneous abortion, premature delivery, abnormal placenta, pre-eclampsia, post-partum haemorrhage, etc., have also been observed, ${ }^{32,33}$ and the rate of caesarean section has also increased. Therefore, for females considering future pregnancy, UAE should be carefully considered for its impact after treating fibroids or adenomyosis. ${ }^{28}$

\section{COMPARISION WITH MEDICAL TREATMENT}

There is no long-lasting, effective medical treatment to reduce the size of uterine fibroids, including short interval treatment with ulipristal. Hence it is not advisable to use medical treatment alone in the management of pressure symptoms secondary to uterine fibroids.

On the other hand, there are many existing reports showing that after HIFU ablation treatment for fibroids and adenomyosis there was no adverse impact on fertility, pregnancy, and labour outcomes.

\section{Impact on Fertility}

There has been concern that HIFU ablation might affect the ovarian function or fertility reserve. Lee et al. ${ }^{34}$ compared the anti-Müllerian hormone $(\mathrm{AMH})$ levels of 70 patients with symptomatic adenomyosis and uterine fibroids before and 6 months after HIFU ablation. They found no significant difference in $\mathrm{AMH}$ levels between the two treatment groups, suggesting that HIFU ablation has minimal impact on ovarian reserve. No AMH changes after HIFU ablation was also confirmed by Cheung et al. ${ }^{35}$ in their study. Therefore, it appears that HIFU ablation is comparatively safer for the fertility of patients receiving treatment.

So far, there has not been a study that demonstrates the impact of HIFU sonication, particularly for a submucosal fibroid and its adjacent endometrial tissue, causing any impairment of implantation. As a result of indirect sonication, the ultrasound energy may impair uterine functions. Direct and excessive ablation of a submucous fibroid might also cause intracavitary adhesions or denuded endometrial lining. Nevertheless, despite a lack of case reports, this possibility cannot be excluded. 


\section{Impact on Pregnancy}

In the early days of magnetic resonanceguided (MRg)-HIFU treatment, case reports of pregnancies showed successful vaginal delivery at term, and none had complications during pregnancy and labour. ${ }^{36,37}$ Some larger studies, including individual and multicentre collaborative trials, have also confirmed successful pregnancies after HIFU ablation for fibroids and adenomyosis. ${ }^{38-41}$

As many studies were from China, the authors had reported a high rate of induced abortions and miscarriage in pregnancies after HIFU treatment for fibroids and adenomyosis. However, it might be related to the fertility regulation in the past. It is also possible that maternal age and sizable fibroids after HIFU may also influence the miscarriage rate in any study, as both factors can independently adversely impact miscarriages. Bohlmann et al. ${ }^{42}$ showed that the risk of miscarriage after ultrasoundguided (USg)FUS/MRgFUS was $17.8 \%$, which did not appear to be higher than a control group of patients wanting to have children.

\section{Impact on Delivery}

From the literature, normal pregnancies have been reported 3-5 months after HIFU treatment. ${ }^{43,44}$ These patients had uneventful vaginal deliveries. There was no uterine rupture during pregnancy or labour for those with vaginal delivery at term after HIFU treatment. Therefore, it appears from these limited data that pregnancy can occur within 1 year after HIFU treatment and a good pregnancy outcome is possible. Conception is advisable 6 months after HIFU.

HIFU delivers pulsed HIFU to the target lesion of uterine fibroid, whereas myomectomy aims to remove the target uterine fibroid. After myomectomy, caesarean section is needed if the uterine cavity has been entered during surgery.

Despite the widespread reassurance that vaginal delivery is safe, the rate of elective caesarean section appeared to be high among females after HIFU ablation. The caesarean section rate was reported as high as $50-78 \% .38,44$ After HIFU ablation, all pregnancies that reach term would have a high caesarean section rate compared to term pregnancy without HIFU surgery. The reasons for the high caesarean section rate are not known; this might be due to a maternal preference.

\section{CONCLUSION}

There is currently no systematic study on the effects on fertility, pregnancy, and delivery outcome for patients after HIFU ablation treatment for fibroids and adenomyosis, but there have been a large number of cases of pregnancy and related reports, suggesting that there is little effect after HIFU on pregnancy. The number of patients requiring continued childbirth is still small, and there is not enough scientific evidence to explain the impact on mother and child during pregnancy.

The current consensus among HIFU doctors, but with limited evidence, suggests that USgFUS/MRgFUS treatment for fibroids and adenomyosis could be a safe alternative to myomectomy or UAE for females who wish to have babies. It also appears that this non-invasive HIFU treatment can shorten the treatment to pregnancy interval after HIFU ablation. Given the prevalence of females with fibroids and adenomyosis before childbearing, addressing these important reproductive issues in a large clinical trial is critical.

\section{References}

1. Mirzamoradi $M$ et al. Evaluation of pregnancy outcomes in Iranian women with uterine myomas. Novelty in Biomedicine. 2020;8(3):142-5.

2. Serour AG. Adenomyosis a forgotten cause of infertility. The Egyptian Journal of Fertility of Sterility. 2020;24(2):11-21.
3. Lee HJ et al. Contemporary management of fibroids in pregnancy. Rev Obstet Gynecol. 2010;3(1):20-7.

4. Mehla S, Singh N. Assessment of pregnancy outcome in patients with uterine fibroids. JAMDSR. 2020;8(11):145-8

5. Harada $T$ et al. The impact of adenomyosis on women's fertility. Obstet Gynecol Surv. 2016;71(9):55768.

6. Halvorson LM. New perspectives on adenomyosis. Semin Reprod Med. 2020;38(2-03):87-8.

7. Szubert $\mathrm{M}$ et al. Adenomyosis and Infertility-review of medical and 
surgical approaches. Int J Environ Res Public Health. 2021;18(3):1235.

8. Xue $\mathrm{M}$ et al., Ultrasound scanning Skil for Adenomyosis: Adenomyosis Facts and treatments (2021) $1^{\text {st }}$ edition, Singapore: Springer, p.70.

9. Wong $\mathrm{F}$ et al. (eds.), Focused Ultrasound Surgery in Gynecology (2021) $1^{\text {st }}$ edition, Singapore: Springer.

10. Maloney E, Hwang JH. Emerging HIFU applications in cancer therapy. Int $J$ Hyperthermia. 2015;31(3):302-9.

11. Huang $L$ et al. High-intensity focused ultrasound combined with dilatation and curettage for caesarean scar pregnancy. Ultrasound Obstet Gynecol. 2014;43(1):98-101.

12. Yang $T$ et al. HIFU for the treatment of difficult colorectal liver metastases with unsuitable indications for resection and radiofrequency ablation: a phase I clinical trial. Surgical Endosc. 2020;35(5):1-10.

13. Marinova $M$ et al. Improving quality of life in pancreatic cancer patients following high-intensity focused ultrasound (HIFU) in two European centers. Eur Radiol. 2021;31(8):1-12.

14. Ziglioli F et al. Oncologic outcome, side effects and comorbidity of high-intensity focused ultrasound (HIFU) for localised prostate cancer. A review. Ann Med Surg (Lond). 2020;56:110-5.

15. Shui L et al. High-intensity focused ultrasound (HIFU) for adenomyosis: two-year follow-up results. Ultrason Sonochem. 2015;27:677-81.

16. Marinova M et al. Novel non-invasive treatment with high-intensity focused ultrasound (HIFU). Ultraschall Med. 2016;37(1):46-55.

17. Li $X$ et al. High-intensity focused ultrasound in the management of adenomyosis: long-term results from a single center. Int J Hyperthermia. 2021:38(1):241-7.

18. Lyon $\mathrm{P}$ et al. Ultrasound-guided high intensity focused ultrasound ablation for symptomatic uterine fibroids: preliminary clinical experience. Ultraschall Med. 2020;41(5):550-6.

19. Shu Li J et al. Pregnancy outcomes in nulliparous women after ultrasound ablation of uterine fibroids: a singlecentral retrospective study. Sci Rep. 2017;7(1):3977.

20. Huang YF et al. A comparison of reproductive outcomes of patients with adenomyosis and infertility treated with high-intensity focused ultrasound and laparoscopic excision. Int J Hyperthermia. 2020;37(1):301-7.

21. Nishiyama $\mathrm{S}$ et al. High recurrence rate of uterine fibroids on transvaginal ultrasound after abdominal myomectomy in Japanese women. Gynecol Obstet Invest. 2006;61(3):155-9.

22. Chen $\mathrm{J}$ et al.; Committee of the ClinicalTrial of HIFU versus Surgical Treatment for Fibroids. Evaluation of high-intensity focused ultrasound ablation for uterine fibroids: an IDEAL prospective exploration study. BJOG. 2018:125(3):354-64

23. Khalilzadeh $\mathrm{O}$ et al. Proposal of a new adverse event classification by the society of interventional radiology standards of practice committee. J Vasc Interv Radiol. 2017;28(10):1432-7.

24. Chen J et al. Safety of ultrasoundguided ultrasound ablation for uterine fibroids and adenomyosis: a review of 9988 cases. Ultrason Sonochem. 2015;27:671-6.

25. Felix Wong WS et al. Adenomyosis - is a new treatment solution available? Clin Exp Obstet Gynecol. 2021;48(1):5-8.

26. Wang F et al. Ultrasound-guided high-intensity focused ultrasound vs laparoscopic myomectomy for symptomatic uterine myomas. J Minim Invasive Gynecol. 2014;21(2):279-84

27. The Hong Kong College of Obstetricians and Gynaecologists (HKCOG). Guidelines for the management of uterine leiomyoma. 2009. Available at: https://www. hkcog.org.hk/hkcog/Download/ Guidelines_for_the_Management_of Uterine_Leiomyoma_2009.pdf. Last accessed: 8 November 2021.

28. de Bruijn AM et al. Uterine artery embolisation for the treatment of adenomyosis: a systematic review and meta-analysis. J Vasc Interv Radiol. 2017;28(12):1629-42.

29. Spies, J.B., et al., Uterine artery embolisation for leiomyomata. Obstet Gynecol. 2001. 98(1):29-34.

30. Spies JB et al. Complications after uterine artery embolization for leiomyomas. Obstet Gynecol. 2002;100(5):873-80.

31. Toor SS et al. Complication rates and effectiveness of uterine artery embolisation in the treatment of symptomatic leiomyomas: a systematic review and metaanalysis. AJR Am J Roentgenology. 2012;199(5):1153-63.

32. Spies JB et al. Ovarian function after uterine artery embolisation for leiomyomata: assessment with use of serum follicle stimulating hormone assay. J Vasc Interv Radiol. 2001;12(4):437-42

33. Pron $\mathrm{G}$ et al. Pregnancy after uterine artery embolisation for leiomyomata: the Ontario multicenter trial. Obstet Gynecol. 2005;105(1):67-76.
34. Lee JS et al. Ultrasound-guided high-intensity focused ultrasound treatment for uterine fibroid \& adenomyosis: a single center experience from the Republic of Korea. Ultrason Sonochem. 2015;27:682-7.

35. Cheung $\vee Y$ et al. Ovarian reserve after ultrasound-guided high-intensity focused ultrasound for uterine fibroids: preliminary experience. J Obstet Gynaecol Can. 2016;38(4):35761.

36. Hanstede MM et al. Focused ultrasound surgery of intramural leiomyomas may facilitate fertility: a case report. Fertil Steril. 2007;88(2):497

37. Morita $Y$ et al. Pregnancy following MR-guided focused ultrasound surgery for a uterine fibroid. Int $J$ Gynecol Obstet. 2007;99(1):56-7.

38. Rabinovici J et al. Pregnancy outcome after magnetic resonanceguided focused ultrasound surgery (MRgFUS) for conservative treatment of uterine fibroids. Fertil Steril. 2010.93(1):199-209.

39. Zou M et al. Pregnancy outcomes in patients with uterine fibroids treated with ultrasound-guided highintensity focused ultrasound. BJOG. 2017:124(3):30-5.

40. Liu $X$ et al. Vaginal delivery outcomes of pregnancies following ultrasoundguided high-intensity focused ultrasound ablation treatment for uterine fibroids. Int J Hyperthermia. 2018;35(1):510-7.

41. Wang $Z$ et al. Observation on pregnancy outcomes after high intensity focused ultrasound ablation therapy in patients with adenomyosis Chongqing Medicine. 2017;36:2506-8.

42. Bohlmann MK et al. High-intensity focused ultrasound ablation of uterine fibroids-potential impact on fertility and pregnancy outcome. Geburtshilfe Frauenheilkunde. 2014;74(02):139-45.

43. Kim KA et al. Short-term results of magnetic resonance imagingguided focused ultrasound surgery for patients with adenomyosis: symptomatic relief and pain reduction. Fertil Steril. 2011.95(3):1152-

44. Rabinovici J et al. Pregnancy and live birth after focused ultrasound surgery for symptomatic focal adenomyosis: a case report. Hum Reprod. 2006.21(5):1255-9.

45. Shu Li J et al. Pregnancy outcomes in nulliparous women after ultrasound ablation of uterine fibroids: a singlecentral retrospective study. Sci Rep. 2017;7(1):3977. 